\title{
RECOVERY VOLTAGE MEASUREMENT ON OIL-PAPER INSULATION WITH SIMPLE GEOMETRY AND CONTROLLED ENVIRONMENT
}

\author{
Y. C. Kuang, G. Chen and P. Jarman \\ School of Electronics and Computer Science \\ University of Southampton, UK \\ National Grid Transco, UK \\ E-mail : yck00r@ecs.soton.ac.uk
}

\begin{abstract}
A theoretical description of recovery voltage on oil-paper insulation systems based on interfacial charge movements is proposed as an alternative to traditional RC-circuit model. The traditional model produces responses that are linear superposition of exponential functions. However the recovery voltage observed in actual time-domain measurement does not conform to the exponential descriptions. This is especially true to the tail of recovery voltage which decay sub exponentially at the rate of $\frac{1}{\sqrt{t}}$ rather than exponentially. More data points need more time constants to describe because the curve is intrinsically non-exponential. The interfacial charge model also suggests that the recovery voltage of pressboard can be viewed as reflecting two major processes of charge movements and transfer.
\end{abstract}

\section{INTRODUCTION}

The problem of interpretation of recovery voltage measurements (RVM) is still an unsolved problem among engineers using the method as a diagnostic tool to probe the health of the power transformers. In an attempt to understand the underlying physical processes that are responsible for producing recovery voltage in oil-paper insulation a series of experiments have been performed. The results of these experiments are reported in [1]-[2]. The changes of recovery voltage with respect to oil moisture level, temperature and sample geometry cannot be explained in consistent manner using traditional Debye polarisation model. Therefore an attempt is made here to propose an alternative physical process that is responsible for the formation of recovery voltage.

\section{Summary of Observations}

Comparing the measured recovery voltage of dry paper in [1] with oil-paper in [2], it is interesting to notice that the peak of recovery voltage can be detected easily on dry pressboard paper but becomes too fast and weak to be detected when the insulating oil is present. The recovery voltage only reappears in oil-paper system when the sample is cut in such a way that charge movements between electrodes is impeded. This suggests that the recovery voltage may be due to interfacial charge transfer and charge diffusion on the surface of paper rather than bulk polarisation through dipole reorientation as commonly believed.
The resistance of paper and oil varies greatly depending on the temperature, moisture level and electric field strength. However from the space charge measurement of oil-paper in [3]we know that even with the electric field strength a thousand time stronger than what is used in recovery voltage measurement the charge migrate through only about twenty micrometers deep in an hours. Therefore we can conclude that the recovery voltage phenomenon is predominantly a surface charge phenomenon on oil-paper insulation. In addition to that we also know from [3] that the charge induced is homocharge. Indirect evidence that charge plays more important role in polarisation of paper than dipole reorientation comes from the dielectric spectroscopy measurements[4]-[5] where low frequency dispersion is the most dominant feature and loss peak is barely discernible. Furthermore the loss peak occurs between $1 \mathrm{~Hz}$ and $10 \mathrm{~Hz}$, most responses in recovery voltage measurement are too slow to be affected.

When the recovery voltage of oil-paper insulation is viewed in time domain one never fails to notice that the tail of the recovery voltage is disproportionately heavy comparing with the discharge curve of linear capacitor. This is especially clear when the charging time is very short as shown in Fig. 1. The voltage decays according to power law rather than exponential law.

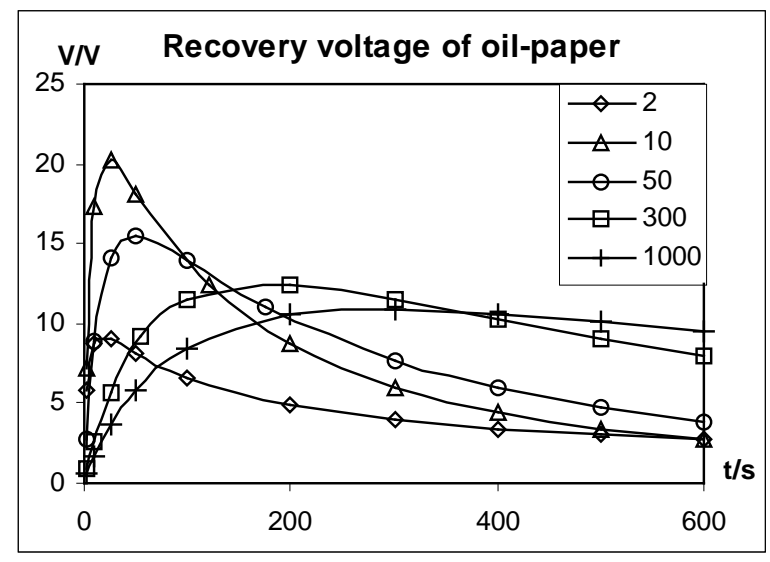

Fig. 1: Recovery voltage of oil-paper pressboard

Another important observation worth pointing out is that the recovery voltage is not strictly repeatable if the relaxation time is not long enough. Depending on temperature, moisture level and whether the paper is immerse in oil the average time scale for getting good 
repeatable recovery voltage is to have relaxation time of about one day between consecutive measurements. This implies weak non-linearity of the mechanism forming recovery voltage. There is still no firm conclusion on the reason for non-linearity but the most obvious possibility is charge trapping by the insulation system. However in order not to obscure the big picture the model that follows in the next section is a linear model for its simplicity to manipulate and interpret.

\section{MODEL}

\section{Charge Transfer model of Recovery Voltage}

Fig. 2 shows a simplified linear circuit approximation of the electrode and the surface of paper. The capacitors directly beneath the electrode will have little interaction because they all experience the same charging and discharging conditions. The capacitors at the edge and beyond will have charge transfer among themselves because the electrode only charge and discharge through the component at the edge of electrode.

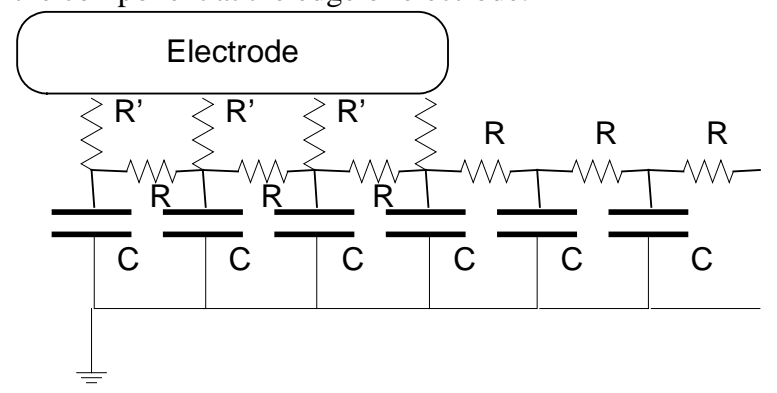

Fig.2: Circuit representation of the oil-paper surface

The difference in voltage between the capacitors at the centre and the capacitors at the edge may cause some charge flow but let us ignore that exchange and assume that the system can be divided into two main groups. The first group is directly beneath the electrode and has the response of the form of exponential decay during discharge. The second group is the chain of capacitors beyond the electrode. The voltage is exerted through charge that is near the edge of the electrode assuming that the voltage of charge far away from electrode has negligible effect on measured voltage. Assuming that the side chain is infinitely long it can be shown that the impedance of the chain is given by (1)

$Z=\frac{R}{2}+\sqrt{\frac{R^{2}}{4}+\frac{R}{s C}}$

where s represents the variable on Laplace space. The presence of Laplace variable under the square roots sign indicates that the resulting voltage to current relationship is non-linear. The reason being the definition of impedance relates the voltage to the flux of charge (current) at one end of the chain but not all charge passing to the capacitor at the end goes into increasing its voltage. Some of the charge actually keeps travelling down the chain to fill up other capacitors. Therefore the chain of linear components actually produces non-linear relation at the end of the chain.

Large number of circuit components is needed in order to model the behaviour of the side chain realistically. One simplification that we can do here is actually taking the limit of infinitesimal small capacitors and resistors. This approximation leads to well known linear diffusion equation with boundary source (2)

$\frac{\partial V}{\partial t}=c \frac{\partial^{2} V}{\partial x^{2}}+f(t) \delta(x)$

The function $f(t)$ in (2) denotes the exchange of charge between electrode and the end of the chain, $\delta(x)$ denotes delta function and $\mathrm{c}$ denotes the diffusion coefficient.

Therefore the simplest model for the measured voltage of such system consists of 3 components: $V_{1}$ the voltage due to capacitors directly beneath the electrode, $V_{2}$ the voltage due to the presence of charge at the end of side chain of capacitors and $V_{e}$ the voltage due to charge on the electrode.

\section{Active Supply Connected}

The value of $\mathrm{V}_{\mathrm{e}}$ is controlled by the voltage supply so that $\mathrm{V}_{\mathrm{s}}=\mathrm{V}_{\mathrm{e}}+\mathrm{V}_{1}+\mathrm{V}_{2}$ is always true throughout this period. Lets the length of charging period be denoted by $t_{c}$ and the length of discharging period be $t_{d}$. The supply voltage $V_{s}$ and its Laplace transform are given by (3). Unless stated otherwise, the same symbol will be used to denote the same function in time domain and Laplace domain because the two seldom appear simultaneously in the same equation.

$$
\begin{aligned}
& V_{s}(t)=V_{0}\left(1-H\left(t-t_{c}\right)\right) \\
& V_{s}(s)=V_{0}\left(\frac{1-\exp \left(-s t_{c}\right)}{s}\right)
\end{aligned}
$$

The solution of $\mathrm{V}_{1}$ can be obtained easily by solving (4). $\beta_{1}$ denotes the rate parameter and $1 / \beta_{1}$ is normally called the time constant of the system. $\alpha_{1}$ denotes the appropriate scaling of the supply voltage to the maximum voltage that can be hold by the capacitors. The value of $\alpha_{1}$ depends on the area of electrode and the permittivity of the sample.

$$
\begin{aligned}
& \frac{d V_{1}}{d t}=-\beta_{1}\left(\alpha_{1} V_{s}-V_{1}\right) \\
& V_{1}(s)=\alpha_{1} V_{0} \frac{\beta_{1}\left(1-\exp \left(-s t_{c}\right)\right)}{s\left(s+\beta_{1}\right)} \\
& V_{1}(t)=\alpha_{1} V_{0}\left(1-\exp \left(-\beta_{1} t_{c}\right)\right) \exp \left(-\beta_{1} t\right)
\end{aligned}
$$

Solving (4) using the Laplace transform method and use (3) to eliminate $V_{s}(s)$ we get the expression for $V_{1}$ in (5). (6) shows $V_{1}$ in time domain for $t>t_{c}$ This is just a normal charging-discharging function of a linear capacitor as expected. 
Rewrite (2) as (7) assuming the current is proportional to the difference between $\mathrm{V}_{\mathrm{s}}$ and $\mathrm{V}_{2}$. Because the space dimension has arbitrary unit $\mathrm{c}$ has been normalised to 1 here. $\alpha_{2}$ and $\beta_{2}$ play similar role as $\alpha_{1}$ and $\beta_{1}$ in (4). Assume that the charge diffuse on one-dimensional space with infinite span and the boundary at $\mathrm{x}=0$ is impermeable. The charge transferred between electrode and paper is modelled as "creation" or "destroy" at the boundary.

$$
\frac{\partial V_{2}}{\partial t}=\frac{\partial^{2} V_{2}}{\partial x^{2}}+\beta_{2}\left(\alpha_{2} V_{s}-V_{2}\right) \delta(x)
$$

These assumptions allow us to use classical Green's function to solve the partial differential equation and obtain an analytic solution.

$$
\begin{aligned}
& V_{2}(x, t)=\int_{0}^{t} \frac{\exp \left(-\frac{x^{2}}{4(t-\tau)}\right)}{\sqrt{\pi(t-\tau)}} \cdot \beta_{2}\left(\alpha_{2} V_{s}-V_{2}(0, \tau)\right) d \tau \\
& \Rightarrow V_{2}(0, t)=\int_{0}^{t} \frac{\beta_{2}\left(\alpha_{2} V_{s}-V_{2}(0, \tau)\right)}{\sqrt{\pi(t-\tau)}} d \tau
\end{aligned}
$$

Lets $\mathrm{P}$ be the Laplace transform of $\mathrm{V}_{2}(0, \mathrm{t})$. Taking Laplace transform of (8) and solve for $\mathrm{P}$ we arrive at (9)

$$
\begin{aligned}
& P=\frac{\beta_{2} \alpha_{2} V_{0}\left(1-\exp \left(-s t_{c}\right)\right)}{s\left(\sqrt{s}+\beta_{2}\right)} \\
& V_{2}(0, t)=\alpha_{2} V_{0}\left[\operatorname{erfcx}\left(\beta_{2} \sqrt{t-t_{c}}\right)-\operatorname{erfcx}\left(\beta_{2} \sqrt{t}\right)\right]
\end{aligned}
$$

The function $\operatorname{erfcx}: x \rightarrow \exp \left(x^{2}\right) \operatorname{erfc}(x)$ is called the scaled complementary error function. It is monotonically decreasing function on real axis approaching $(x \sqrt{\pi})^{-1}$ asymptotically. However we need the whole charge distribution as the initial condition for calculation during the measurement phase. Substitute $\mathrm{V}_{2}(0, \mathrm{t})$ back into the original expression involving $\mathrm{V}_{2}(\mathrm{x}, \mathrm{t})$ and solve the convolution through Laplace transform method we get the charge distribution as a function of both space and time in (10).

$$
V_{2}(x, t)=\alpha_{2} V_{0}\left[g(x, t)-g\left(x, t-t_{c}\right)\right]
$$

The function $\mathrm{g}(\mathrm{x}, \mathrm{t})$ is given below:

$$
g(x, t)=\operatorname{erfc}\left(\frac{x}{\sqrt{4 t}}\right)-\exp \left(-\frac{x^{2}}{4 t}\right) \operatorname{erfcx}\left(\frac{x}{\sqrt{4 t}}+\beta_{2} \sqrt{t}\right)
$$

Evaluating $\mathrm{V}_{2}(\mathrm{x}, \mathrm{t})$ at $\mathrm{t}=\mathrm{t}_{\mathrm{c}}+\mathrm{t}_{\mathrm{d}}$ will give us the charge distribution just before the measurement is initiated.

\section{Measurement}

Reset the time reference to zero when the measurement started to simplify the following discussions and since we have all the initial conditions this does not distort the end results.

$$
\begin{aligned}
& \left.V_{1}(0)=\alpha_{1} V_{0} 1-\exp \left(-\beta_{1} t_{c}\right)\right) \exp \left(-\beta_{1} t_{d}\right) \\
& V_{2}(0,0)=\alpha_{2} V_{0}\left(\operatorname{erfcx}\left(\beta_{2} t_{d}\right)-\operatorname{erfcx}\left(\beta_{2}\left(t_{c}+t_{d}\right)\right)\right)
\end{aligned}
$$

$V_{e}(0)=-V_{1}(0)-V_{2}(0,0)$

Without the power supply attached to electrode $V_{e}$ is now free to interact with $\mathrm{V}_{1}$ and $\mathrm{V}_{2}$. Assume that the charge exchange between $\mathrm{V}_{\mathrm{e}}, \mathrm{V}_{1}$ and $\mathrm{V}_{2}$ are proportional to their difference the following set of simultaneous differential equations describe the dynamics of the variables. $\eta$ denotes overall loss of charge to the surrounding and the measurement system.

$$
\begin{gathered}
\frac{d V_{e}}{d t}=-\beta_{1}\left(\alpha_{1} V_{e}-V_{1}\right)-\beta_{2}\left(\alpha_{2} V_{e}-V_{2}\right)-\eta V_{e} \\
\frac{d V_{1}}{d t}=\beta_{1}\left(\alpha_{1} V_{e}-V_{1}\right) \\
V_{2}(0, t)=\int_{0}^{t} \frac{\beta_{2}\left(\alpha_{2} V_{e}(\tau)-V_{2}(0, \tau)\right)}{\sqrt{\pi(t-\tau)}} d \tau \\
+\int_{0}^{\infty} \frac{\exp \left(-\frac{y^{2}}{4 t}\right)}{\sqrt{\pi t}} V_{2}(y, 0) d y
\end{gathered}
$$

Working in Laplace domain again we can transform the above set of differential equations into the following set of simultaneous equations.

$$
\begin{aligned}
& \left(\begin{array}{ccc}
s+\alpha_{1} \beta_{1}+\alpha_{2} \beta_{2}+\eta & -\beta_{1} & -\beta_{2} \\
-\alpha_{1} \beta_{1} & s+\beta_{1} & 0 \\
-\alpha_{2} \beta_{2} & 0 & \sqrt{s}+\beta_{2}
\end{array}\right)\left(\begin{array}{c}
V_{e} \\
V_{1} \\
V_{2}
\end{array}\right) \\
& =\left(\begin{array}{c}
V_{e}(0) \\
V_{1}(0) \\
\alpha_{2} V_{0}\left(G\left(s, t_{c}+t_{d}\right)-G\left(s, t_{d}\right)\right)
\end{array}\right)
\end{aligned}
$$

The "initial value" of $\mathrm{V}_{2}$ comes from integrating the initial charge distribution in (16) and $\mathrm{G}(\mathrm{s}, \mathrm{T})$. is given by the following equation:

$$
G(s, T)=\frac{1-\operatorname{erfcx}(\sqrt{s T})}{\sqrt{s}}+\frac{\operatorname{erfcx}(\sqrt{s T})-\operatorname{erfcx}\left(\beta_{2} \sqrt{T}\right)}{\sqrt{s}-\beta_{2}}
$$

It can be proved that $\mathrm{G}(\mathrm{s}, \mathrm{T})$ is non-singular at $\sqrt{s}=\beta_{2}$. Although the above system of equations can be inverted analytically, the inverse Laplace transform is performed numerically[6]. Numerical inversion will allow for more complex extension of the model to be developed in the future where analytic inverse transform may not exist. Finally the measured voltage can be obtained by adding all three variables: $V=V_{e}+V_{1}+V_{2}$.

It can be seen that the proposed model is bare minimum where no interaction between $V_{1}$ and $V_{2}$ is included and all the leakage of charge is lumped into one variable $\eta$. Furthermore the charge transfer is assumed to be linear to voltage difference and consideration of charge trapping is excluded. The model may need modification for different boundary conditions. For instance the assumption of infinitely long chain of capacitor without leakage will no longer be true if there exist a charge 
leakage path with small resistance such as the flat disk sample and disk with rim sample mentioned in [2]. The working out of charge distribution is important for the final result but it is a non-trivial exercise and analytic solution may be unattainable if more complex boundary conditions are imposed. The technique of fractional calculus is an attractive alternative over working out the detail of charge distribution. However because the measurement of recovery voltage involves 3 transients from start to end the use of fractional calculus becomes excessively complicated in the end to cope with the changes in boundary conditions.

\section{COMPARISON WITH MEASURED DATA}

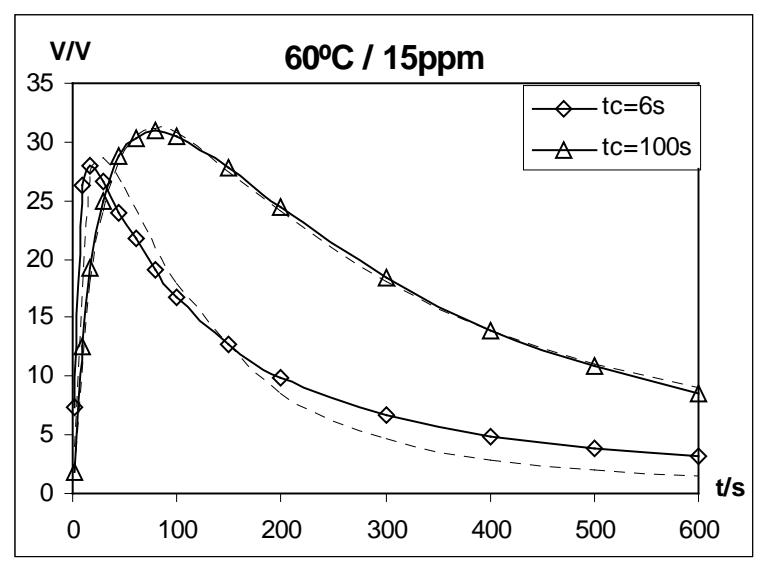

Fig. 3: Comparing model prediction and measured data for $t_{c}=\{6 s, 100 s\}$

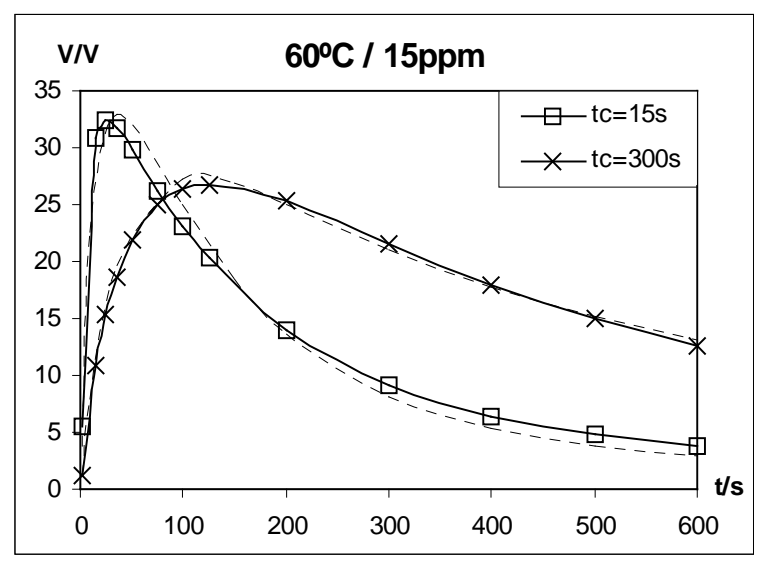

Fig. 4: Comparing model prediction and measured data for $t_{c}=\{15 s, 300 s\}$

The model derived above will need to be tested against measured data to see how well it fits the actual observations. Fig. 3 and Fig. 4 show the model prediction and the measured data using the parameters in Table 1. The data shown in Fig. 3 and Fig. 4 are the recovery voltage measured at $60^{\circ} \mathrm{C}$ and $15 \mathrm{ppm}$ moisture with one electrode covered up by the sample as described in [2]. Solid lines with markers represent the measured recovery voltage while dotted lines represent the calculated values based on the proposed model and parameters in Table 1. Only two rate parameters are needed to describe the data with $t_{c}$ over two orders of magnitude and all parameters have well defined physical interpretation.

\begin{tabular}{|c|c|c|c|c|c|}
\hline Parameter & $\alpha_{1}$ & $\alpha_{2}$ & $\beta_{1}$ & $\beta_{2}$ & $\eta$ \\
\hline Value & 0.1153 & 0.0501 & 0.1076 & 1.5750 & 0.0163 \\
\hline
\end{tabular}

\section{Table 1: Parameter values for Fig. 3 and Fig. 4}

The model works better at higher temperatures possibly because the charge trapping is less significant at higher temperature and makes the charge transfer more linear with respect to voltage difference as in one of the assumptions made. Both the dry paper and oil-paper exhibit certain dependence of history. If this is cause by trapping of charge on the surface of paper, the most important implication is that the value of parameters may be slightly different during charging and discharging period. This has not been taken into account in the model proposed above.

\section{CONCLUSION}

A model that is based on charge transfer on the surface of oil-paper insulation is used to explain the observation that recovery voltage of oil-paper insulation with nonstandard geometry cannot be accounted by commonly used linear circuit approximation.

\section{ACKNOWLEDGEMENT}

The authors would like to thank the directors of National Grid Company plc, UK for permission to publish this paper and financial support for this project.

\section{REFERENCES}

[1] Y.C.Kuang, G.Chen, Recovery voltage measurement on Kraft paper pressboard, $2^{\text {nd }}$ ICMEP Conf., Chongqing, Oct 2003, p 495-498

[2] Y.C.Kuang, G.Chen, Recovery voltage measurement on oil-paper insulation with simple geometry and controlled environment, $8^{\text {th }}$ ICSD Conf., Toulouse, July 2004

[3] P.Morshuis, M.Jeroense, Space charge measurements on impregnated paper: A review of the PEA method and a discussion of results, IEEE Elec. Ins. Magazine, May-Jun 1997, v 13, n 3, p 26-35 [4] Y.Du, M.Zahn, Moisture and temperature effects on the dielectric spectrum of transformer pressboard, $\mathrm{C}$ EIDP Annual Report, 2002, p 878-881

[5] R.Ek, R.M.Hill, Low frequency dielectric spectroscopy characterization of microcrystalline cellulose, tablets and paper, J. Materials Sci., Sep 1997, v 32, n 18, p 4807-4814

[6] F.R.de Hoog, J.H.Knight, A.N.Stokes, An improved method for numerical inversion of Laplace transforms, S.I.A.M. J. Sci. Stat. Comp, 1982 v.3, p 357-366. 